\title{
A Bijection between classes of Fully Packed Loops and Plane Partitions
}

\author{
P. Di Francesco,\# \\ P. Zinn-Justin * \\ and J.-B. Zuber \#
}

Submitted: Nov 21, 2003; Accepted: Sep 5, 2004; Published: Sep 16, 2004

MR Subject Classifications: Primary 05A19; Secondary 52C20, 82B20

\begin{abstract}
It has recently been observed empirically that the number of FPL configurations with 3 sets of $a, b$ and $c$ nested arches equals the number of plane partitions in a box of size $a \times b \times c$. In this note, this result is proved by constructing explicitly the bijection between these FPL and plane partitions.
\end{abstract}

\section{Introduction}

Configurations of Fully Packed Loops, or FPL in short, are sets of disconnected paths visiting once each vertex of a square $n \times n$ grid, and exiting through every other of the $4 n$ external edges. While these FPL constitute an interesting and much studied model of statistical mechanics, they have also attracted recently the attention of combinatorialists by their connections with alternating sign matrices, tiling problems, plane partitions and related topics (see for example [1] for an overall review). Moreover, there is a yet mysterious relation with a linear problem: the numbers of FPL configurations of different "link patterns" give the components of the Perron-Frobenius eigenvector of an operator (Hamiltonian) constructed in terms of the generators of the Temperley-Lieb algebra [2].

In a recent work [3], one of us has observed empirically that the number of FPL with 3 sets of $a, b$ and $c$ nested arches, $(a+b+c=n)$, is nothing else than the number

\# Service de Physique Théorique de Saclay, CEA/DSM/SPhT, URA 2306 du CNRS, C.E.A.-Saclay, F-91191 Gif sur Yvette Cedex, France; philippe@spht.saclay.cea.fr zuber@spht.saclay.cea.fr

* Laboratoire de Physique Théorique et Modèles Statistiques, UMR 8626 du CNRS, Université Paris-Sud, Bâtiment 100, F-91405 Orsay Cedex, France; pzinn@lptms.upsud.fr 
of plane partitions in a box of size $a \times b \times c$ (MacMahon formula)

$$
\begin{aligned}
P P(a, b, c) & =\frac{\left(\begin{array}{c}
n-1 \\
a
\end{array}\right)\left(\begin{array}{c}
n-2 \\
a
\end{array}\right) \cdots\left(\begin{array}{c}
n-b \\
a
\end{array}\right)}{\text { same for } n=a+b} \\
& =\prod_{i=1}^{a} \prod_{j=1}^{b} \prod_{k=1}^{c} \frac{i+j+k-1}{i+j+k-2} \\
& =\frac{H(a+b+c) H(a) H(b) H(c)}{H(a+b) H(b+c) H(c+a)} .
\end{aligned}
$$

In the third of these equivalent expressions, $H$ is the hyperfactorial function $H(p)=(p-$ $1) !(p-2) ! \cdots 1$ !. It is the object of this note to prove this result by constructing explicitly the bijection between these FPL and plane partitions. In fact a similar bijection between FPL with different boundary conditions and a tiling problem had been constructed by de Gier [4], and our construction is closely related to his method.

We now state precisely the result. Let us consider a FPL with three sets of nested arches; let $a, b, c$ be the numbers of nested arches; and $A, B$ and $C$ be the centers of the three bundles of arches, namely the central unoccupied external edges. We call such a FPL a "FPL of type $(A, B, C)$ ". The number of occupied external edges between $A$ and $B$ is $a+b$. Thus the data of $A, B, C$ determine $a, b, c$.

ThEOREM. There is a bijection between FPL configurations of type $(A, B, C)$ (with a link pattern made of three nested sets of $a, b$ and $c$ arches) and the plane partitions in a box of size $a \times b \times c$.

\section{The bijection}

In this section, we explicitly construct the bijection between FPL configurations of type $(A, B, C)$ and plane partitions in a box of sides $a, b, c$.

Given the center $A$ of a bundle of arches, on the side of the $n \times n$ square, we construct the cone which is the space between the two diagonals at 45 degrees starting from the innermost endpoint of the unoccupied edge $A$. One then proves easily that there exists a unique point among $A, B, C$ such that its cone contains the two others; this point is never in a corner. Up to a permutation of the letters $A, B, C$ (and of the associated $a, b, c)$, we may always assume that this point is $C$ and then, three cases may occur (see Fig. 1 for the generic case, and 2 for a sample of typical cases that we shall follow throughout this paper):

(i) $A$ and $B$ lie on the same side of the square, which is necessarily opposite to $C$, since otherwise $a+c$ or $b+c \geq n$, which is absurd;

(ii) $A$ lies on the side opposite to $C$, and $B$ on an adjacent side: any such configuration or its mirror image is as depicted on Fig. $1(\mathrm{ii})$;

(iii) $A$ and $B$ are on sides adjacent to that of $C$, which are necessarily opposite.

Remark: the limiting cases where $A$ and/or $B$ is in a corner offer no difficulty and are treated as in the general case. 


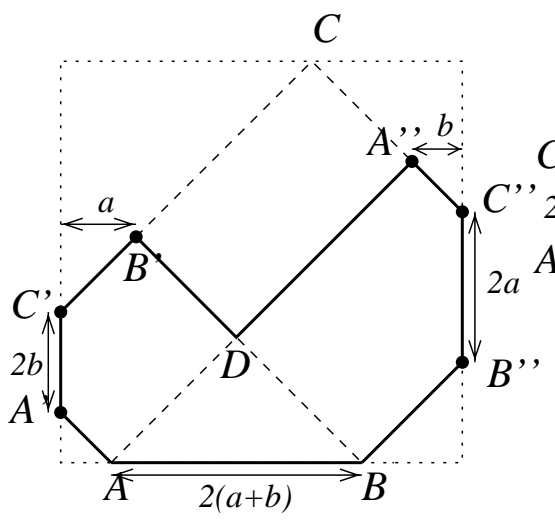

(i)

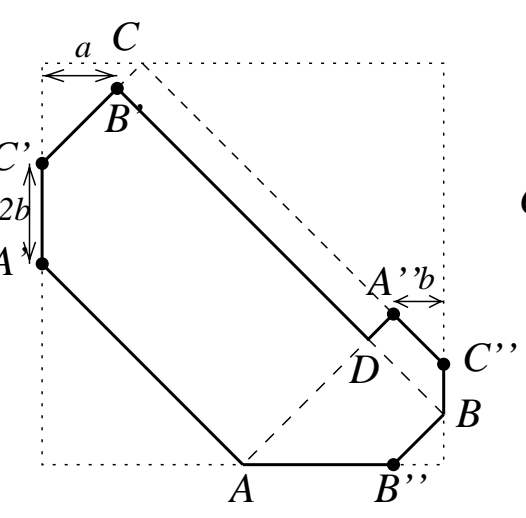

(ii)

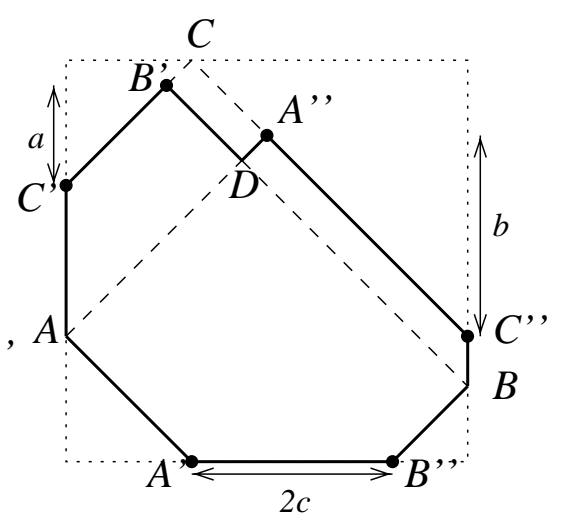

(iii)

Fig. 1: The three generic cases, with $A$ and $B$ in the cone of $C$ : $(i) A, B$ on the opposite side of $C$; $(i i) A$ on the opposite side of $C$ and $B$ on an adjacent one; (iii) $A$ and $B$ on distinct adjacent sides to $C$. In each case, we have represented the polygon $P^{\prime}$ in thick black lines. The distances shown on the figure equal the number of elementary segments, possibly rounded to one of the nearest integers.

In all that follows, we shall treat in parallel these 3 cases; even though the reasoning is essentially the same, there are some technical differences between the 3 situations.

We now describe the 4 steps required to produce the bijection, postponing to the next section the actual proof of the theorem.

Step 1 From the points $C$, draw the diagonals (lines of slope \pm 1 ; they are the boundaries of the cone mentioned above), which cross the sides of the square at $C^{\prime}, C^{\prime \prime}$. From $A$, resp. $B$, draw also the diagonals, and call $A^{\prime}$ and $A^{\prime \prime}$, resp. $B^{\prime}$ and $B^{\prime \prime}$, their intersection with a side of the square or with the diagonals coming from $C$, whichever comes first. (Some of these points may coincide). Finally let $D$ be the intersection of $A A^{\prime \prime}$ and $B B^{\prime}$. Let $P$ be the polygon $\left(A A^{\prime}\right) C^{\prime} C C^{\prime \prime}\left(B^{\prime \prime} B\right)$, where the brackets $\left(A A^{\prime}\right)$ mean a pair up to transposition, depending on the case (see Fig. 1 and 2); and $P^{\prime}$ be the polygon $\left(A A^{\prime}\right) C^{\prime} B^{\prime} D A^{\prime \prime} C^{\prime \prime}\left(B^{\prime \prime} B\right)$.

Step 2 Pave the inside of the polygon $P^{\prime}$ with dominos, in the way indicated on Fig. 2 (note that in all cases the polygon is not quite filled with the dominos due to diagonal lines and in the vicinity of $D$ ).

Step 3 One can deform the dominos into a subset of the hexagonal lattice. One can check, see Fig. 2, that in the three cases this subset has the desired shape of a hexagon with sides of lengths $a, b, c$. The middle edges of the dominos become diameters of the hexagons.

Step 4 Finally, to each FPL of type $(A, B, C)$, associate a dimer configuration on the subset of the hexagonal lattice by keeping only the FPL edges of the borders of the dominos, while discarding all other edges including the middle edges of the dominos; and finally deform the dominos into the hexagons as explained in step 3. The dimer configuration can equivalently be represented as a plane partition in a 


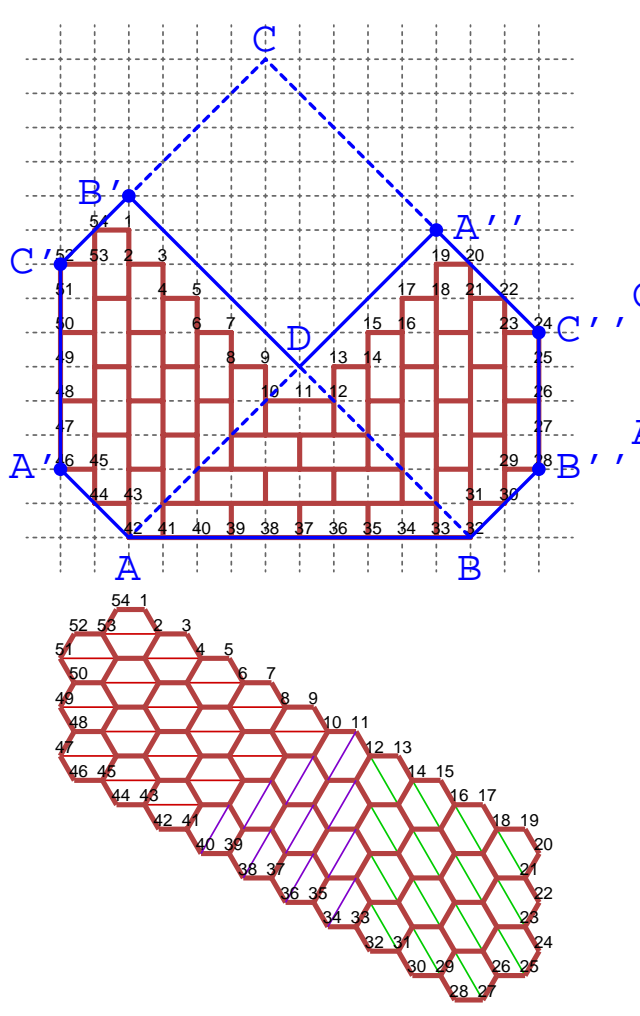

(i)
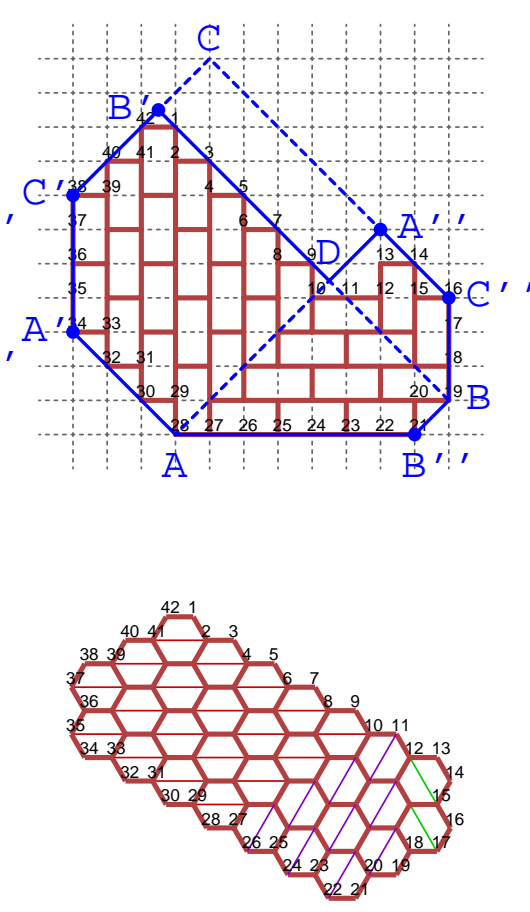

(ii)
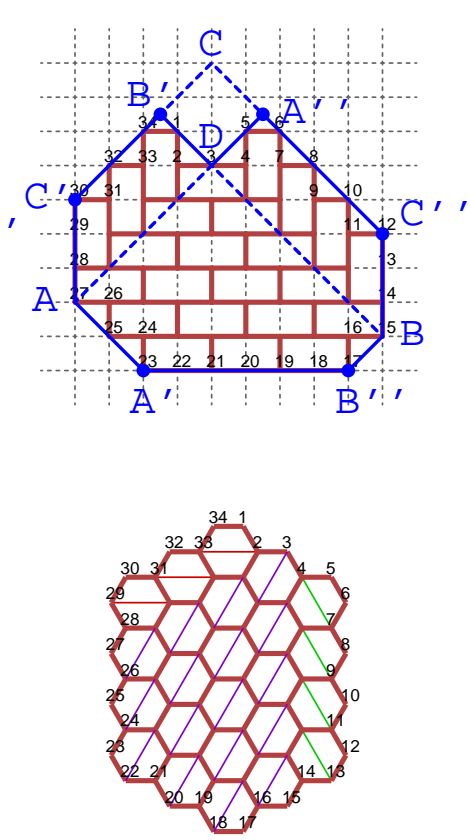

(iii)

Fig. 2: Polygons, domnios and hexagons. The values of $(a, b, c)$ are respectively $(2,3,10),(3,2,7)$ and $(3,4,3)$.

box $a \times b \times c$, see Fig. 3 .

\section{Proof of the Theorem}

An important part of the proof is devoted to the determination of the fixed edges, i.e. the edges which are occupied (or unoccupied) in any configuration of the given type $(A, B, C)$. We first recall a very useful lemma proved by de Gier [4]

Lemma 1. In Fig. 4, if (i) the edges $a b$ and ef are occupied, with $a b$ and ef belonging to different loops, and if (ii) $c d$ either is an unoccupied external edge, or belongs to a third loop, or is connected to ab by da or to ef by de, then the edge $k l$ is occupied.

Proof of lemma 1: if $k l$ was not occupied, either both $b k$ and $k f$ would, which would contradict (i), or $d k$ and either $k b$ or $k f$ would, which would contradict (ii).

Lemma 2. The edges outside the polygon $P^{\prime}$, as well as some edges inside the polygon, are fixed as depicted on Fig. 7.

Proof of lemma 2: we shall build the fixed edges in 2 steps (cf Fig. 5-7).

1. We first prove that in each triangular domain limited by one of the diagonals starting from $A, B$ or $C$ and the sides of the square, and exterior to the polygon $P$, the edges are fixed and form "stairs". The external edges incident to such a triangle connect to 

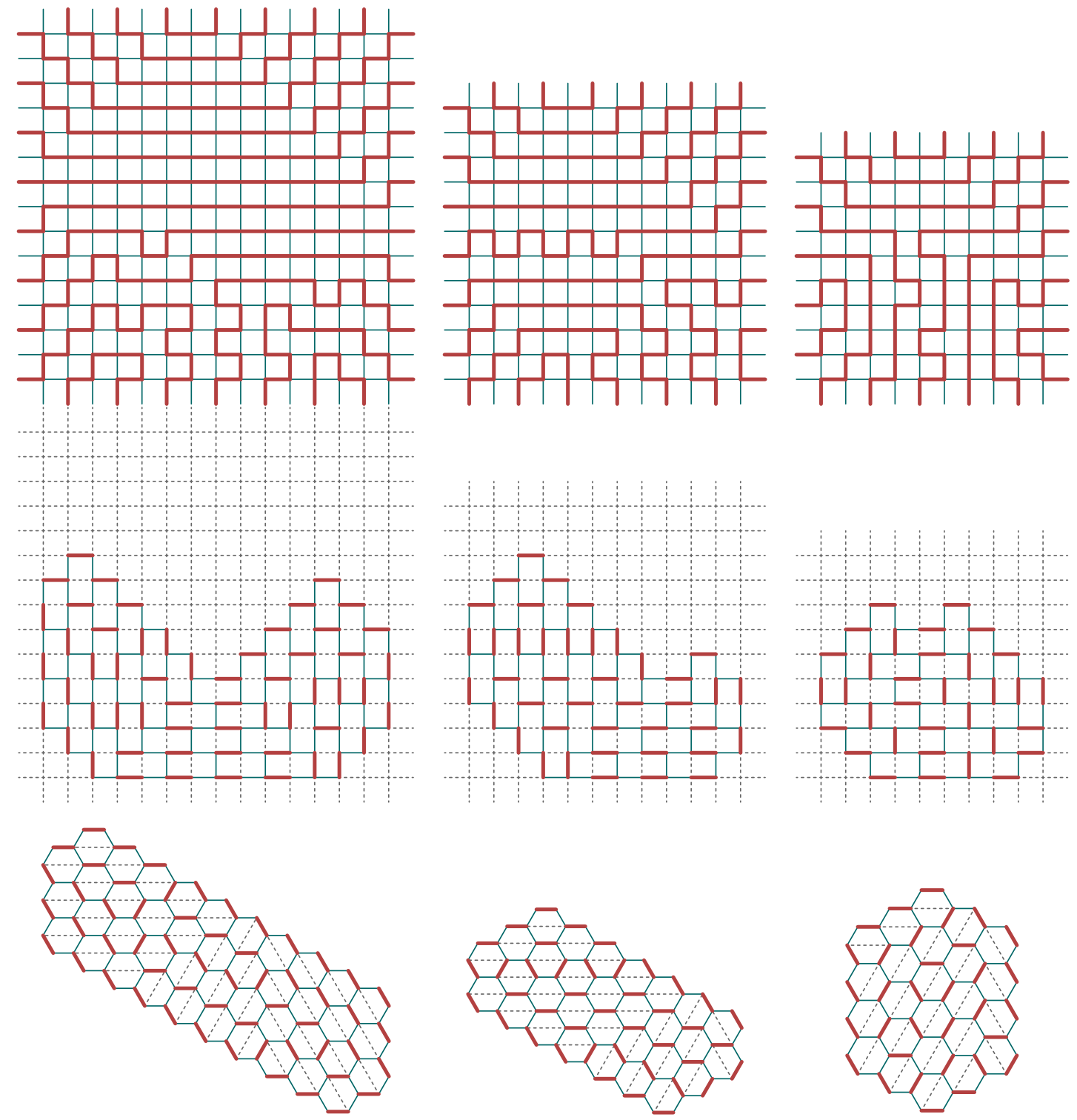

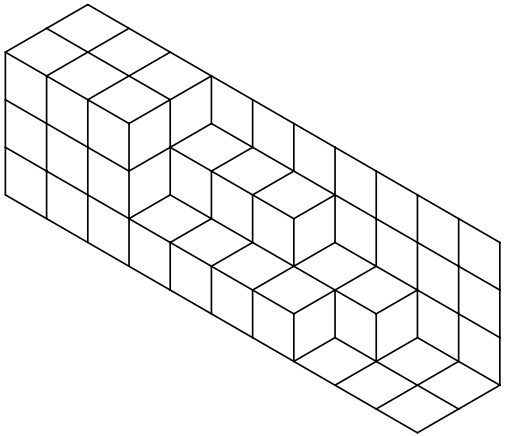

(i)

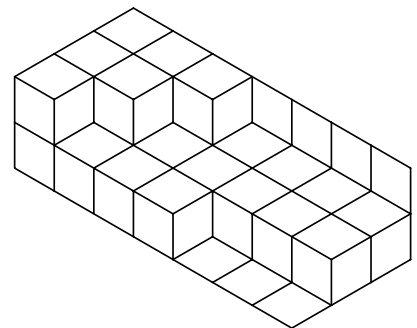

(ii)

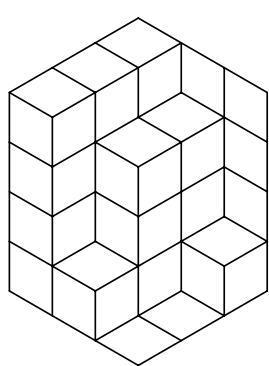

(iii)

Fig. 3: The bijection: examples. 


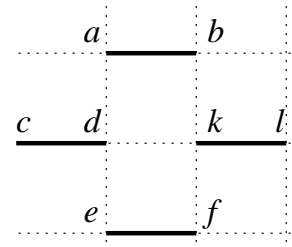

Fig. 4: de Gier's lemma

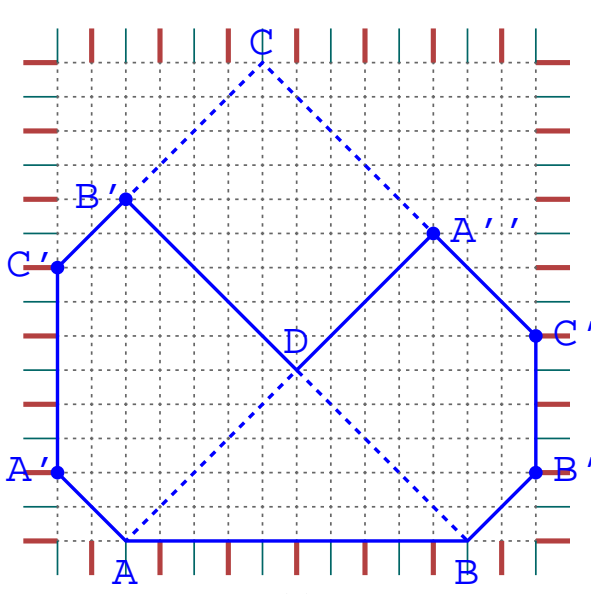

(i)

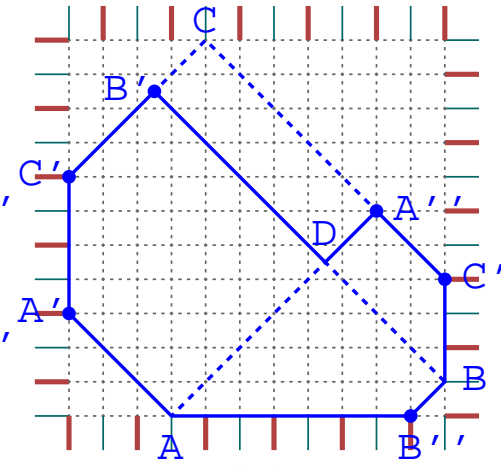

(ii)

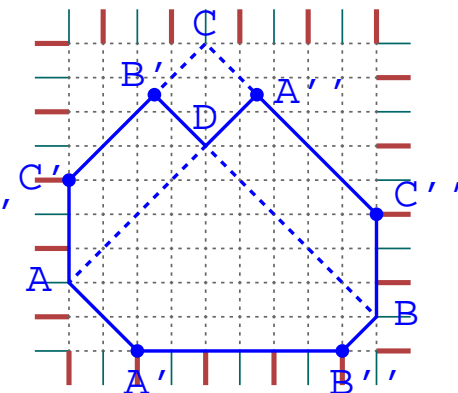

(iii)

Fig. 5: Empty grids with the polygon drawn.

external edges that are on the other side of this diagonal and the "loops" that start from them must thus cut the diagonal at distinct points. As there are as many points on this diagonal as there are external edges, the only possibility is the stair pattern.

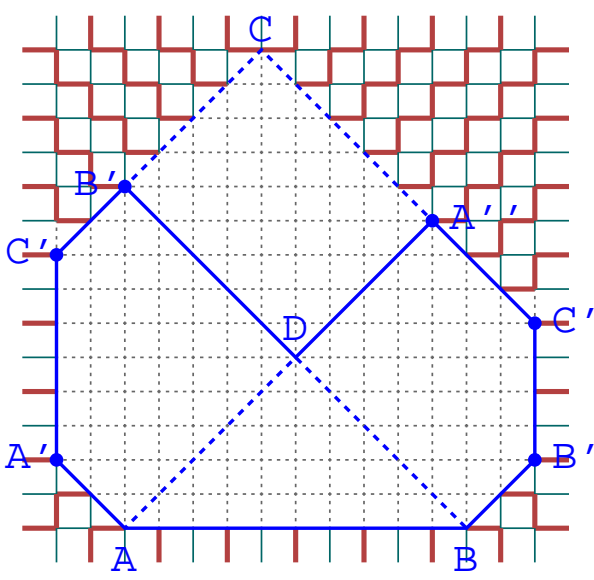

(i)

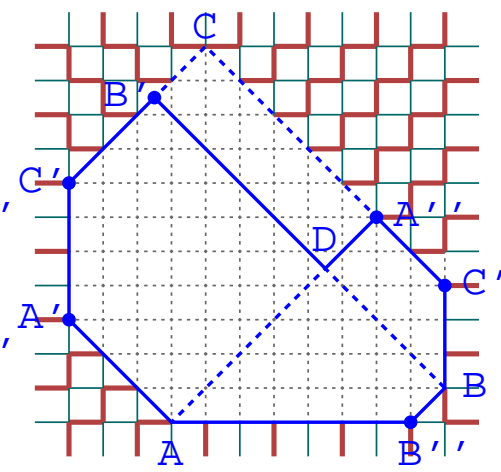

(ii)

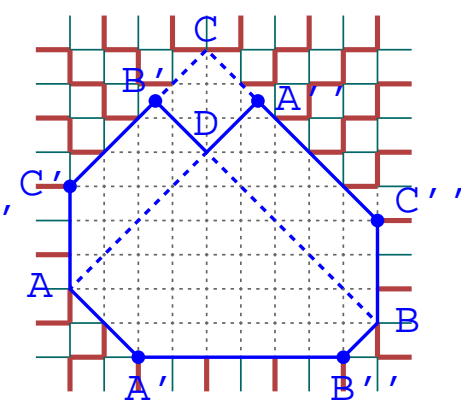

(iii)

Fig. 6: Fixed edges outside the polygon, either occupied (thick lines) or unoccupied (thin lines). 
2. Edges inside the polygon may also be fixed by repeated action of lemma 1, starting from the sides of the square. The fact that all the external edges that lie on the same side of any of the diagonals belong to different loops enables one to iterate the application of lemma 1. While this lemma fixes every other edge in a given direction (horizontal or vertical), all horizontal edges in the rectangle $C B^{\prime} D A^{\prime \prime}$ are fixed, by a successive application of the argument starting from the left and from the right external edges.

Note that at this stage, each vertex of the square grid belongs to at least one fixed edge. At the vertices belonging to two occupied edges, the complementary edges are also fixed to be unoccupied (thin solid lines on Fig. 7). Those vertices which belong to only one internal fixed edge may be regarded as active, two or three unfixed lines emanate from them (dashed lines on Fig. 7) and it could be possible to switch to a dual picture, by depicting their dual triangles and looking at the various ways one may assemble them into lozenges, etc. This is the route followed by de Gier [4] and Krattenthaler [5].

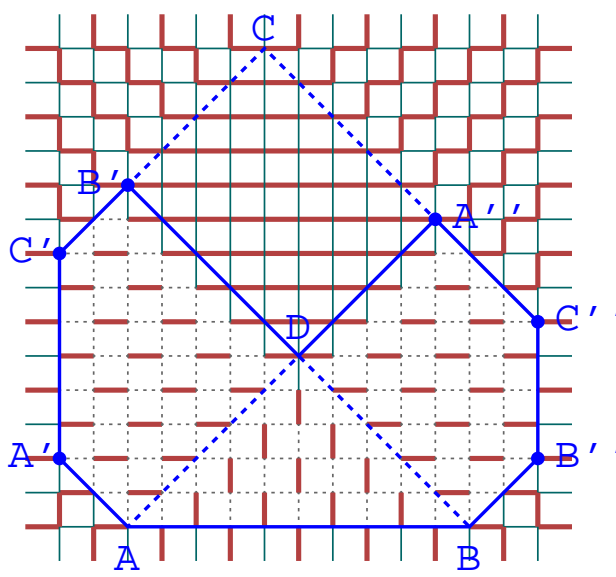

(i)

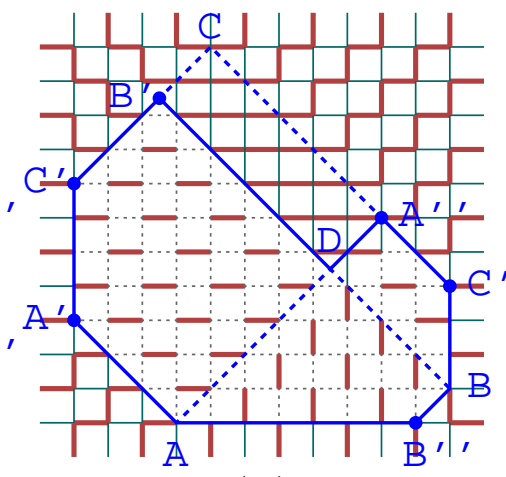

(ii)

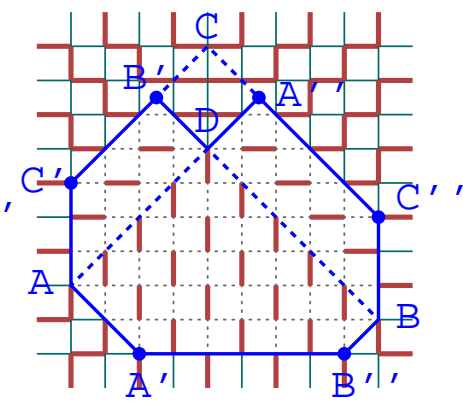

(iii)

Fig. 7: The full set of fixed edges.

Lemma 3. There exists a FPL configuration of type $(A, B, C)$. It is loopless.

Proof of lemma 3: one may construct explicitly the two special FPL configurations which are mapped onto the empty or the full plane partition. Here we shall describe the construction for one of the two, and we let the reader repeat it for the other. For this purpose, we carry out a further splitting of the domains of our grid, and construct the points $T, E, H, H^{\prime}$ and $H^{\prime \prime}$ (see Fig. 8). In each of the domains limited by the polygon $P^{\prime}$ and by dashed lines, the yet unfixed edges are determined according to the indicated prescription (in red). It is now easy to see that the $a$ loops entering the grid between $A$ and $H$ will exit through $A H^{\prime}$, and likewise the $b$ loops entering in $B H$ exit through $B H^{\prime \prime}$ and the $c$ loops entering in $C H^{\prime \prime}$ exit through $C H^{\prime}$. This is illustrated on Fig. 8, in which examples of staircase loops are represented in each of the domains bounded by the dotted (green) lines (see also Fig. 9 for the pictures in our three typical examples). We thus have constructed explicitly a configuration of type $(A, B, C)$, and it has no closed loop. Note that the point $E$ in our construction has an interpretation 


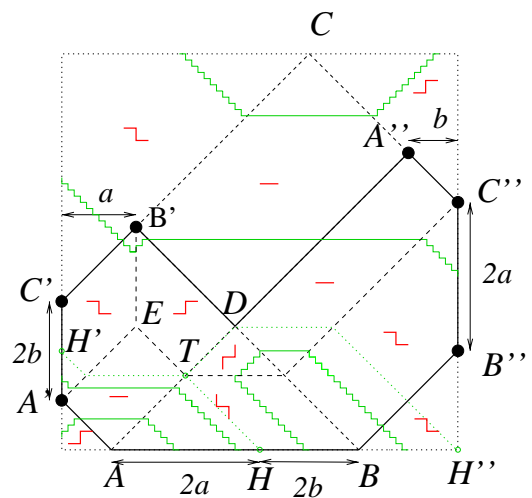

(i)

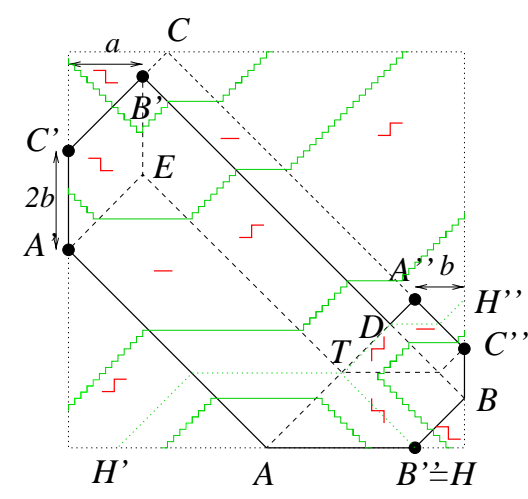

(ii)

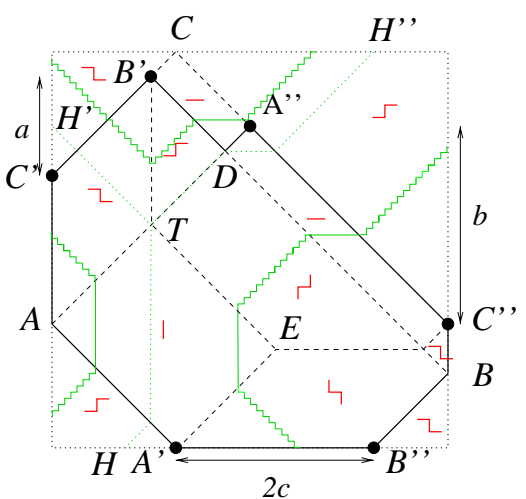

(iii)

Fig. 8: FPL configurations corresponding to the "empty" plane partition: the generic case.

in the FPL $\leftrightarrow$ plane partition matching: it corresponds to the corner of the (say empty) box.

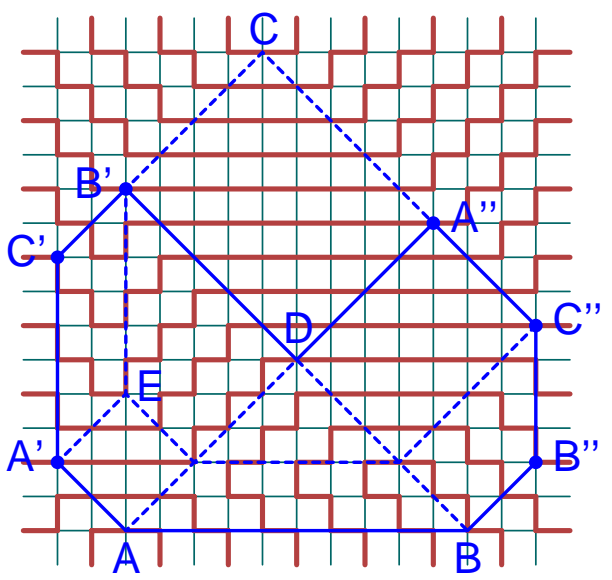

(i)

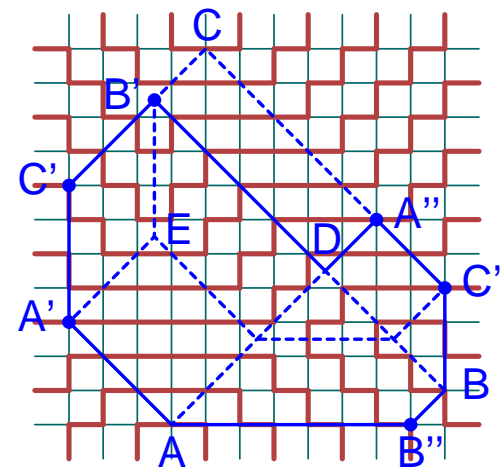

(ii)

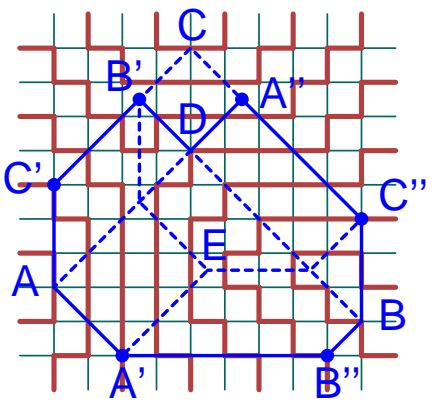

(iii)

Fig. 9: FPL configurations corresponding to the "empty" plane partition in our three examples.

Proof of theorem: we now have all the elements to complete the proof of the theorem. Let us go back to the construction of section 2, step 4. To a FPL of type $(A, B, C)$ we associate the configuration obtained by restriction to the dominos inside the polygon $P^{\prime}$. Note that the edges of the dominos are exactly the complementary set of the fixed edges of Fig. 7; therefore lemma 2 implies that this mapping is injective. By simple inspection one can check that in all three cases depicted on Fig. 2, the dominos can be deformed into an $(a, b, c)$ hexagon. Furthermore, since exactly two edges around each vertex are occupied on the original square lattice, and the middle edges of the dominos are fixed to be occupied, the new configuration on the hexagonal lattices is precisely 
a dimer configuration, or in the dual language, a tiling of the $(a, b, c)$ hexagon with lozenges, or still equivalently, a plane partition in a box of size $a \times b \times c$.

We thus have an injective mapping from FPL of type $(A, B, C)$ into plane partitions. To prove its surjectivity, we note the following. The moves elementary hexagons of plane partitions, or equivalently the moves $\square \leftrightarrow \square$ on dimer configurations of the hexagonal lattice are well-known to be ergodic, namely allow to explore the full set of plane partitions in an $a \times b \times c$ box. (This ergodicity is most conspicuous in the equivalent picture of cube stackings in a box of size $a \times b \times c$.) In the correspondence above, an elementary hexagon becomes deformed into a domino, and once the fixed middle edge is added, the move becomes $\square \leftrightarrow \square \square$ or $\square \leftrightarrow \square$. A key remark is that this move does not modify the connection between the 4 corners of the domino; hence, it preserves the link pattern of the whole configuration (and does not add or remove any loops). Starting from a particular configuration, which we choose to be the one exhibited in lemma 3 , one can produce using such moves the preimage of any plane partition. Therefore the mapping is surjective.

Alongside this proof, we have established two corollaries:

Corollary 1. The moves $\square \leftrightarrow \square$ and $\square \square \square \square$ are ergodic on FPL configurations of type $(A, B, C)$.

Corollary 2. There are no internal loops in any FPL configuration of type $(A, B, C)$.

Both properties had been stated by de Gier [4] but no detailed proof had been given.

\section{Concluding comments}

The reader who is not yet fully convinced is invited to visit the site http://ipnweb.in2p3.fr/lptms/membres/pzinn/fpl

to practice a little and to enjoy the show!

Since plane partitions come with a natural grading (the number of boxes) (up to the ambiguity with the complement), our bijection yields a grading of FPL of type $(A, B, C)$ and Macdonald formula $\prod_{i=1}^{a} \prod_{j=1}^{b} \prod_{k=1}^{c} \frac{1-q^{i+j+k-1}}{1-q^{i+j+k-2}}$ gives the relevant counting. It would be nice to have a direct interpretation of this grading in the language of FPL, and to see if it extends to other types of FPL.

By Wieland's theorem [6], it is known that the number of FPL of a given link pattern is invariant under the action of the dihedral group $D_{2 n}$ on this link pattern. One observes empirically that the effect of a Wieland's rotation is to rotate the diameters of the elementary hexagons of step 3 in sec. 2 (more precisely, the point $D$ which determines the three zones of diameters, see Fig. 2, turns around the boundary of the hexagon 4 vertices at a time), while the dimer configuration is left invariant. In other words, a FPL configuration and its Wieland rotation share the same image under the respective bijections. Note that Wieland's rotations do not create any loop in this case, even though they do in general. 
Note that the two problems connected by our bijection, namely the FPL counting and the plane partition counting, may both be rephrased in terms of the 6-vertex model. They correspond however to different weights and to different fixed boundary conditions.
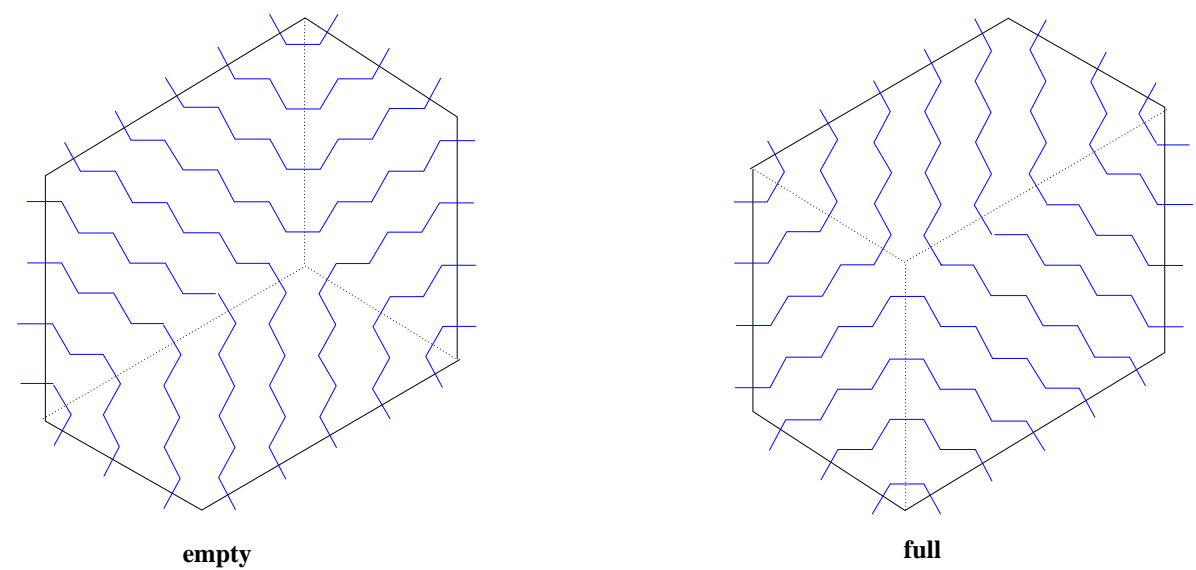

Fig. 10: Full/empty configurations in the HFPL picture, for $(a, b, c)=$ $(5,4,3)$.

A last remark is in order. The dimer formulation of rhombus tilings or plane partitions in an $a \times b \times c$ box may also be rephrased by considering the "loops" formed by the edges complementary to the dimers on the underlying hexagonal lattice. These loops are paths that connect points on the perimeter of the $(a, b, c)$ hexagon and form Fully Packed Loop configurations of the hexagonal lattice, referred to as HFPL. The HFPL are nothing but the images of the complement of the FPL of type $(A, B, C)$ in our bijection. As is readily seen, these complementary loops connect every other point on the perimeter of the square to every point on the perimeter of the domino tiling of the active zone (i.e. the polygon $P^{\prime}$ ), via "parallel" paths. Thus, our bijection also yields a one-to-one mapping of the FPL's complement to the HFPL, and in particular the link patterns of the latter yield those of the former. Among the HFPL two are particularly simple: they correspond to the two fundamental states of an empty or a completely filled plane partition, see Fig. 10. As the HFPL paths must travel along parallel zig-zag lines on the three visible sides of the (empty or filled) box, their link pattern is also of the type $(a, b, c)$, and so is that of the two corresponding FPL's complements, which therefore have no internal loop. The complement of a generic FPL of type $(a, b, c)$, however, may have internal loops, as the basic move does not preserve the connectivity of the complementary edges. The identification with HFPL provides therefore yet another grading of FPL, according to the number of internal loops of the complement or equivalently of the associated HFPL. Conversely, this allows to view all HFPL of an $(a, b, c)$ hexagon as the complement of the restriction of FPL to $(a, b, c)$ link patterns. One may wonder whether more general FPL's complements could correspond to HFPL on more general domains. 


\section{Acknowledgments}

It is a pleasure to thank J. de Gier, J. Propp and D. Wilson for stimulating comments and especially C. Krattenthaler for informing us of his own work on proving the bijection and for fruitful exchanges. This work is partially supported by the European networks HPRN-CT-2002-00325 and HPRN-CT-1999-00161.

\section{References}

[1] J. Propp, The many faces of alternating-sign matrices, in "Discrete Models: Combinatorics, Computation, and Geometry" (special issue of Discrete Mathematics and Theoretical Computer Science), July 2001, (math.C0/0208125).

[2] A.V. Razumov and Yu.G. Stroganov, Combinatorial nature of ground state vector of O(1) loop model, Theor.Math.Phys. 138 (2004) 333-337; Teor.Mat.Fiz. 138 (2004) 395-400, (math.C0/0104216).

[3] J.-B. Zuber, On the Counting of Fully Packed Loop Configurations. Some new conjectures, Electron. J. Combin. 11 (2004) \# R13, (math-ph/0309057).

[4] J. de Gier, Loops, matchings and alternating-sign matrices, Proceedings of the 14th International Conference on Formal Power Series and Algebraic Combinatorics (Melbourne 2002), to appear in Discr. Math., preprint (math.C0/0211285).

[5] C. Krattenthaler, private communication.

[6] B. Wieland, A large dihedral symmetry of the set of alternating-sign matrices, Electron. J. Combin. 7 (2000) R37, (math.C0/0006234). 